\title{
Subjet Multiplicities at LHC Energies and the QCD Color Factor Ratio $C_{A} / C_{F}$
}

\author{
M. Kaur and Anter P. Kaur \\ Department of Physics, Panjab University, Chandigarh 160014, India \\ Correspondence should be addressed to M. Kaur; manjit@pu.ac.in
}

Received 22 August 2013; Revised 3 November 2013; Accepted 5 November 2013

Academic Editor: Kingman Cheung

Copyright (C) 2013 M. Kaur and A. P. Kaur. This is an open access article distributed under the Creative Commons Attribution License, which permits unrestricted use, distribution, and reproduction in any medium, provided the original work is properly cited.

Subjet multiplicity distributions of two jets with highest transverse momenta are simulated in proton-proton collisions at LHC energies of $7 \mathrm{TeV}$ and $14 \mathrm{TeV}$. Jets are defined via the software package FastJet version 3.0.3 which includes the implementation of $k_{T}$ sequential recombination algorithm with a jet size of $R=0.6$. Subjets are resolved by rerunning the $k_{T}$ algorithm on the jets and using the resolution cutoff of $y_{\text {cut }}=10^{-3}$. The subjet multiplicity distributions and their average values are measured as a function of the jet momenta and upto rapidities $|\eta|<2$. The results are used to calculate the ratio, $r=\left(\left\langle M_{g}\right\rangle-1\right) /\left(\left\langle M_{q}\right\rangle-1\right)$.

\section{Introduction}

One of the main motivations of studying highly energetic proton-proton (p-p) collisions at Large Hadron Collider (LHC) at CERN has been the discovery of Higgs Boson(s). The aim has recently been achieved with the discovery of a Higgs Boson. However, many more studies are needed to establish its properties. In addition, searches for new particles will continue for many more years.

The proton-proton collisions at the LHC are dominated by jet production which constitute a large background to potential signals of new physics. Quantum chromodynamics (QCD) describes well the dynamics of jet production in terms of partons. In QCD, quarks and gluons have different color charges and hence different coupling strengths. The values for the color factors originate directly from the symmetry group $\mathrm{SU}(3)$ of $\mathrm{QCD}$, calculated to be $C_{A}=3$ for the gluon and $C_{F}=4 / 3$ for the quark [1]

In hadron-hadron collisions, quarks and gluons fragment to produce collimated streams of particles conventionally called "jets." A jet, characterized by its energy and momentum, is reconstructed according to a clustering prescription called a "jet algorithm" from the four vectors of constituents that may represent tracks, energy deposits in the calorimeter, reconstructed particle candidates, particles in a Monte Carlo
(MC) event generator, or partons of a theory calculation. In dijet events, the two jets leading in transverse momentum $P_{T}$ can be associated with the two partons at leading order (LO) in perturbative QCD. The internal structure of these jets is then expected to depend mainly on the type of the primary parton, that is, either (anti-) quark or gluon, from which they originated. QCD predicts that gluons, because of their larger color factor, fragment more than quarks. Consequently, gluon-initiated jets become broader and exhibit a larger constituent multiplicity than quark jets. The ratio of the constituent multiplicity for gluon jets versus quark jets at LO is asymptotically given by the ratio of their color factor as $C_{A} / C_{F}=9 / 4$. Effects of higher orders in the strong coupling constant $\alpha_{S}$, however, could change this theoretical prediction.

In order to study the jet substructure, an experimental observable called subjet multiplicity, $M$, is found to be very useful. It is defined as the number of subjets that can be resolved within a jet by reclustering the jet constituents with the same clustering algorithm. However, the spatial resolution is chosen to be finer than the one used in the jet reconstruction. In this way an infrared- and collinear-safe measure can be defined that is usable experimentally as well as in perturbative QCD. Various collinear and infrared safe jet algorithms exist [2]. 
TABLE 1: Average subjet multiplicities for jets matched to gluons and quarks in the $|\eta|$ bins with $P_{T}^{\text {jet }}>100 \mathrm{GeV}$ for $k_{T}$ algorithm at $\sqrt{s}=7 \mathrm{TeV}$ and $\sqrt{s}=14 \mathrm{TeV}$.

\begin{tabular}{|c|c|c|c|c|c|c|c|c|}
\hline \multirow{3}{*}{$|\eta|$} & \multicolumn{4}{|c|}{$7 \mathrm{TeV}$} & \multicolumn{4}{|c|}{$14 \mathrm{TeV}$} \\
\hline & \multicolumn{2}{|c|}{ Gluon jets } & \multicolumn{2}{|c|}{ Quark jets } & \multicolumn{2}{|c|}{ Gluon jets } & \multicolumn{2}{|c|}{ Quark jets } \\
\hline & $\langle M\rangle$ & Stat. & $\langle M\rangle$ & Stat. & $\langle M\rangle$ & Stat. & $\langle M\rangle$ & Stat. \\
\hline$|\eta|<1.0$ & 3.39 & \pm 0.01 & 2.27 & \pm 0.01 & 3.44 & \pm 0.01 & 2.27 & \pm 0.01 \\
\hline $1.0 \leq|\eta|<2.0$ & 3.46 & \pm 0.01 & 2.26 & \pm 0.01 & 3.45 & \pm 0.01 & 2.32 & \pm 0.01 \\
\hline
\end{tabular}

TABLE 2: Average subjet multiplicities as a function of $|\eta|$ in different $\widehat{p}_{T}$ bins, for jets with $P_{T}^{\text {jet }}>100 \mathrm{GeV}$ for $k_{T}$ algorithm at $\sqrt{s}=7 \mathrm{TeV}$ and $\sqrt{s}=14 \mathrm{TeV}$.

\begin{tabular}{|c|c|c|c|c|c|}
\hline \multirow{2}{*}{$\widehat{p}_{T}(\mathrm{GeV})$} & \multirow{2}{*}{$|\eta|$} & \multicolumn{2}{|c|}{$7 \mathrm{TeV}$} & \multicolumn{2}{|c|}{$14 \mathrm{TeV}$} \\
\hline & & $\langle M\rangle$ & Stat. & $\langle M\rangle$ & Stat. \\
\hline \multirow{4}{*}{$50-300$} & $|\eta|<0.5$ & 2.99 & \pm 0.05 & 3.25 & \pm 0.06 \\
\hline & $0.5 \leq|\eta|<1.0$ & 2.90 & \pm 0.07 & 3.06 & \pm 0.07 \\
\hline & $1.0 \leq|\eta|<1.5$ & 2.89 & \pm 0.09 & 3.14 & \pm 0.08 \\
\hline & $1.5 \leq|\eta|<2.0$ & 2.71 & \pm 0.11 & 3.02 & \pm 0.09 \\
\hline \multirow{4}{*}{$300-550$} & $|\eta|<0.5$ & 2.11 & \pm 0.01 & 2.21 & \pm 0.01 \\
\hline & $0.5 \leq|\eta|<1.0$ & 2.06 & \pm 0.01 & 2.18 & \pm 0.01 \\
\hline & $1.0 \leq|\eta|<1.5$ & 2.01 & \pm 0.01 & 2.17 & \pm 0.01 \\
\hline & $1.5 \leq|\eta|<2.0$ & 1.95 & \pm 0.02 & 2.10 & \pm 0.01 \\
\hline \multirow{4}{*}{$550-800$} & $|\eta|<0.5$ & 1.82 & \pm 0.01 & 1.94 & \pm 0.01 \\
\hline & $0.5 \leq|\eta|<1.0$ & 1.77 & \pm 0.01 & 1.90 & \pm 0.01 \\
\hline & $1.0 \leq|\eta|<1.5$ & 1.70 & \pm 0.01 & 1.86 & \pm 0.01 \\
\hline & $1.5 \leq|\eta|<2.0$ & 1.65 & \pm 0.04 & 1.80 & \pm 0.01 \\
\hline \multirow{4}{*}{$800-1050$} & $|\eta|<0.5$ & 1.68 & \pm 0.01 & 1.81 & \pm 0.01 \\
\hline & $0.5 \leq|\eta|<1.0$ & 1.63 & \pm 0.01 & 1.77 & \pm 0.01 \\
\hline & $1.0 \leq|\eta|<1.5$ & 1.51 & \pm 0.01 & 1.72 & \pm 0.01 \\
\hline & $1.5 \leq|\eta|<2.0$ & 1.49 & \pm 0.08 & 1.64 & \pm 0.02 \\
\hline
\end{tabular}

Following a sequential recombination procedure, jets are defined by $k_{T}$ algorithm which starts clustering first the softest objects, trying to undo the effects of parton showering [3-5] and is well suited to explore the jet substructure. $k_{T}$ algorithm is implemented in the FastJet package [6-8].

In the present work, samples used to measure the subjet multiplicity at two center of mass energies (c.m.), $7 \mathrm{TeV}$ and $14 \mathrm{TeV}$, corresponding to the current and future LHC energies, respectively, are generated from the event generator PYTHIA8 [9]. A sample of 7 million events at each of the two c.m. energies is generated. The dijet events are selected in which the average subjet multiplicity $\langle M\rangle$ is studied as a function of the jet $P_{T}$ in the range of 100 to $1100 \mathrm{GeV}$ and in the pseudorapidity interval $|\eta|<2$, where pseudorapidity $\eta=-\ln \tan (\theta / 2)$ with $\theta$ as the azimuthal angle of the jet.

\section{Jet Algorithm and the Subjet Multiplicity}

The $k_{T}$ algorithm [5] clusters all input objects $i, i=1, \ldots n$, according to the following iterative steps. (i) For each pair $(i, j)$ of objects find

$$
d_{i j}=\min \left(p_{T i}^{2}, p_{T j}^{2}\right) \frac{\Delta R_{i j}^{2}}{R^{2}},
$$

where $R=0.6$ is the jet size in $y-\phi$ space

$$
\Delta R_{i j}=\sqrt{\left(y_{i j}\right)^{2}+\left(\Delta \phi_{i j}\right)^{2}} .
$$

(ii) For each object calculate the beam distance

$$
d_{i b}=p_{T i}^{2}
$$

(iii) If the minimum $d_{\min }$ of all possible $d_{i j}$ and $d_{i b}$ is $d_{i j}$, then merge objects $i$ and $j$ into a single object by 4 -momentum vector addition. If it is $d_{i b}$ then remove object $i$ from the list and define it to be a final jet.

(iv) Repeat the above steps until no clustering objects are left.

2.1. Subjet Multiplicities. Subjets are resolved within a jet by repeating the application of $k_{T}$ cluster algorithm described 
above. The clustering is terminated when all $d_{i j}, d_{i b}$ are above the quantity $d_{\text {cut }}=y_{\text {cut }} P_{T}^{2}$ (jet). All remaining objects are called subjets. While the stopping parameter $d_{\text {cut }}$ defines the hard scale of the process, the parameter $y_{\text {cut }}$ is known as the resolution parameter. The subjet structure depends upon the values chosen for the resolution parameter $y_{\text {cut }}$. The mean subjet multiplicity $\langle M\rangle$ is defined as the average number of subjets in a jet at a given value of $y_{\text {cut }}$ :

$$
\langle M\rangle=\frac{1}{N_{\text {jet }}} \sum_{i=1}^{N_{\text {jet }}} M\left(y_{\text {cut }}\right) .
$$

By definition $M\left(y_{\text {cut }}\right) \geq 1$ and $0 \leq y_{\text {cut }} \leq 1$. The mean subjet multiplicity is measured for $y_{\text {cut }}=10^{-3}$ and $R=0.6$, where $y_{\text {cut }}$ is the subjet resolution parameter. A study to optimise the value of $y_{\text {cut }}$ at $7 \mathrm{TeV}$ was done in an earlier work, documented in $[10,11]$ and the same used here. This optimum value of $y_{\text {cut }}$ matches with the one used for the definition of subjets in [12]. In addition two subjets within a jet are resolved if they are well separated in $\eta \times \phi$ space.

\section{Determination of Ratio $r$}

In the p-p collisions, the final state stable particles are arranged into jets by using a jet algorithm, as described above. These particles are produced in the hadronisation of hard partons. In a mixed sample of quark and gluon initiated particle jets, $M$ is the subjet multiplicity which can be written as a linear combination of subjet multiplicity in gluon jets $M_{g}$ and quark jets $M_{q}$ :

$$
M=f M_{g}+(1-f) M_{q},
$$

where $f$ is the fraction of gluon jets and $(1-f)$ is the fraction of quark jets in the mixed sample. The gluon jet fraction $f$ is the number of outgoing gluons that pass the selection cuts divided by the total number of outgoing partons that pass the selection cuts. Considering the above equation for two similar samples of jets at $\sqrt{s}$ ( $\sqrt{s}$ is the center of mass energy (c.m.)) $=7 \mathrm{TeV}$ and $14 \mathrm{TeV}$ and assuming $M_{g}$ and $M_{q}$ to be independent of $\sqrt{s}$, we get

$$
\begin{aligned}
M^{7} & =f^{7} M_{g}+\left(1-f^{7}\right) M_{q}, \\
M^{14} & =f^{14} M_{g}+\left(1-f^{14}\right) M_{q} .
\end{aligned}
$$

The solutions are

$$
\begin{gathered}
M_{q}=\frac{f^{14} M^{7}-f^{7} M^{14}}{f^{14}-f^{7}}, \\
M_{g}=\frac{\left(1-f^{7}\right) M^{14}-\left(1-f^{14}\right) M^{7}}{f^{14}-f^{7}},
\end{gathered}
$$

where $M^{14}$ and $M^{7}$ are extracted from the total subjets in mixed samples of quark and gluon initiated jets in the data at $\sqrt{s}=14$ and $7 \mathrm{TeV}$, respectively, and $f^{14}$ and $f^{7}$ are the gluon jet fractions at the two energies. This method relies on the extraction of quark and gluon fractions from the Monte Carlo information, obtained by tagging the particle jets with partons. This way the knowledge of the two gluon jet fractions is obtained. The subjet multiplicity distributions can be characterized by the mean values $\langle M\rangle$ such that $\left\langle M_{g}\right\rangle-1$ and $\left\langle M_{q}\right\rangle-1$ gives the average number of subjets in a gluon and quark jet, respectively. The gluon jets are compared to the quark jets by having a ratio:

$$
r=\frac{\left\langle M_{g}\right\rangle-1}{\left\langle M_{q}\right\rangle-1} .
$$

\section{Event Samples and Jet Selection}

To study the subjet multiplicity in high $P_{T}$ jets and to calculate the color factor ratio, events are generated using PYTHIA8 with CTEQ6L1 PDFs at $\sqrt{s}=7 \mathrm{TeV}$ and $14 \mathrm{TeV}$. At each energy, 1 million events are generated in 4 bins of $\widehat{p}_{T}$ in the pseudorapidity range $|\eta|<2$. The bins in $\widehat{p}_{T}$ are $50-300,300-550,550-800$, and $800-1050 \mathrm{GeV}$. One million events are generated in each pseudorapidity range $|\eta|<$ 1 and $1 \leq|\eta|<2$. One million events are also generated separately in each bin of jet pseudorapidity $|\eta|$ which are $0.0-$ $0.5,0.5-1.0,1.0-1.5$, and $1.5-2.0$ for leading jets. Thus in total we have generated 7 million events for each energy for the present study.

Quark and gluon jets are unambiguously defined only in the leading order QCD where there are only two jets, that is, two partons in the final state. So a sample of dijet events in hard QCD $2 \rightarrow 2$ scattering events is defined by selecting the two jets leading in $P_{T}$ in each event with the following selection cuts:

(i) $P_{T}^{\text {jet }}>100 \mathrm{GeV}$,

(ii) $\left|\Delta \phi\left(j_{1}, j_{2}\right)-\pi\right|<1.0$ (requiring the jets to be backto-back in the azimuthal plane),

(iii) $|\eta|<2$.

For $k_{T}$ algorithm, mean subjet multiplicity is measured for cone radius, $R=0.6$ and $y_{\text {cut }}=10^{-3}$.

\section{Discrimination between Quark and Gluons Jets}

To determine the ratio of color factor, jet substructure of gluon and quark initiated jets is studied in terms of subjet multiplicities. For the Monte Carlo PYTHIA8 events used here, the quark and gluon jets are identified using parton level information. The direction of each primary parton is determined after its perturbative evolution has terminated. The jet closest to the direction of the primary parton (quark/antiquark or gluon) tagged to be the quark/gluon jet, respectively. The two highest $P_{T}$ jets are identified as quark or gluon initiated jets via the tagging in the spatial coordinates and the kinematical cuts. Thus we implement these cuts by allowing the maximum distance in $\eta \times \phi$ space as $\Delta R=\sqrt{(\Delta \eta)^{2}+(\Delta \phi)^{2}}<0.4$. The maximum relative 
TABLE 3: Average subjet multiplicities as a function of $P_{T}^{\text {jet }}(\mathrm{GeV})$ for mixed jets and jets matched to gluons and quarks, for $k_{T}$ algorithm at $\sqrt{s}=7 \mathrm{TeV}$ and $\sqrt{s}=14 \mathrm{TeV}$.

\begin{tabular}{|c|c|c|c|c|c|c|}
\hline$P_{T}^{\text {jet }}(\mathrm{GeV})$ & $\langle M\rangle$ & Stat. & $\left\langle M_{g}\right\rangle$ & Stat. & $\left\langle M_{q}\right\rangle$ & Stat. \\
\hline \multicolumn{7}{|c|}{$7 \mathrm{TeV}$} \\
\hline $100-300$ & 2.63 & \pm 0.01 & 3.06 & \pm 0.01 & 2.05 & \pm 0.01 \\
\hline $300-500$ & 2.03 & \pm 0.01 & 2.45 & \pm 0.01 & 1.72 & \pm 0.01 \\
\hline $500-700$ & 1.82 & \pm 0.01 & 2.25 & \pm 0.01 & 1.58 & \pm 0.01 \\
\hline $700-900$ & 1.71 & \pm 0.01 & 2.17 & \pm 0.01 & 1.53 & \pm 0.01 \\
\hline $900-1100$ & 1.64 & \pm 0.01 & 2.11 & \pm 0.01 & 1.49 & \pm 0.01 \\
\hline \multicolumn{7}{|c|}{$14 \mathrm{TeV}$} \\
\hline $100-300$ & 2.76 & \pm 0.01 & 3.07 & \pm 0.01 & 2.13 & \pm 0.01 \\
\hline $300-500$ & 2.11 & \pm 0.01 & 2.43 & \pm 0.01 & 1.70 & \pm 0.01 \\
\hline $500-700$ & 1.93 & \pm 0.01 & 2.28 & \pm 0.01 & 1.60 & \pm 0.01 \\
\hline $700-900$ & 1.82 & \pm 0.01 & 2.17 & \pm 0.01 & 1.56 & \pm 0.01 \\
\hline $900-1100$ & 1.75 & \pm 0.01 & 2.11 & \pm 0.01 & 1.52 & \pm 0.01 \\
\hline
\end{tabular}

TABLE 4: Fraction of gluon and quark jets in phase space $|\eta|<2$ in different $P_{T}^{\text {jet }}(\mathrm{GeV})$ bins for $k_{T}$ algorithm.

\begin{tabular}{lcccccrrr}
\hline \multirow{2}{*}{$P_{T}^{\text {jet }}(\mathrm{GeV})$} & \multicolumn{2}{c}{ Gluon Jets } & \multicolumn{2}{c}{$7 \mathrm{TeV}$} & \multicolumn{2}{c}{ Quark Jets } & \multicolumn{2}{c}{$14 \mathrm{TeV}$} \\
& $f$ & Stat. & $1-f$ & Stat. & $f$ & Stat. & $1-f$ & Stat. \\
\hline $100-300$ & 0.57 & \pm 0.01 & 0.43 & \pm 0.01 & 0.68 & \pm 0.01 & 0.32 \\
$300-500$ & 0.44 & \pm 0.01 & 0.56 & \pm 0.01 & 0.56 & \pm 0.01 & 0.44 \\
$500-700$ & 0.33 & \pm 0.01 & 0.67 & \pm 0.01 & 0.48 & \pm 0.01 & 0.52 \\
$700-900$ & 0.27 & \pm 0.01 & 0.73 & \pm 0.01 & 0.42 & \pm 0.01 & 0.58 \\
$900-1100$ & 0.22 & \pm 0.01 & 0.78 & \pm 0.01 & 0.37 & \pm 0.01 & \pm 0.01 \\
\hline
\end{tabular}
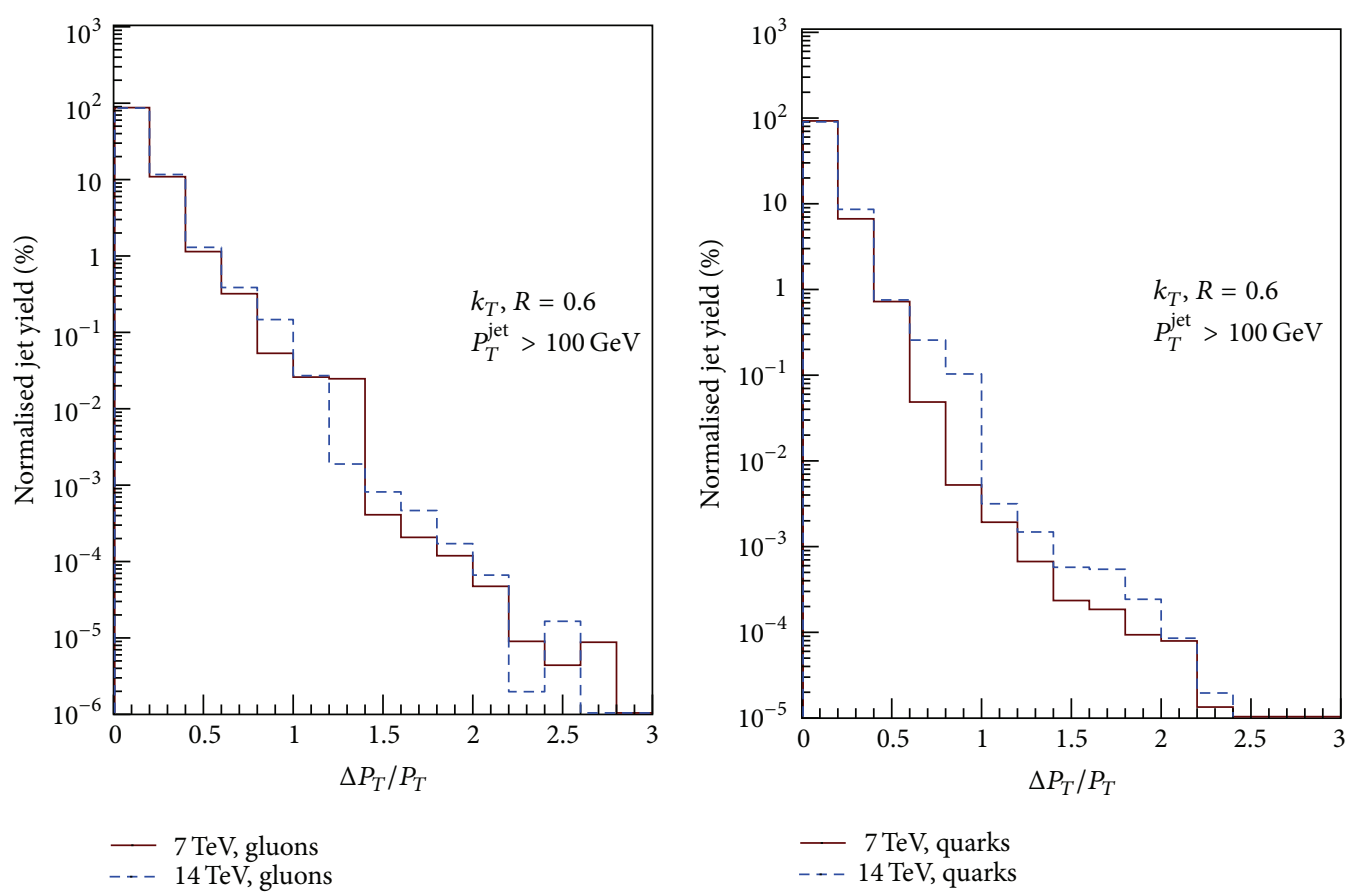

Figure 1: Distribution of $\Delta P_{T} / P_{T}$ for the matched quark and gluon jets with $P_{T}^{\text {jet }}>100 \mathrm{GeV}$ at $\sqrt{s}=7 \mathrm{TeV}$ compared to $\sqrt{s}=14 \mathrm{TeV}$ for $k_{T}$ algorithm. 

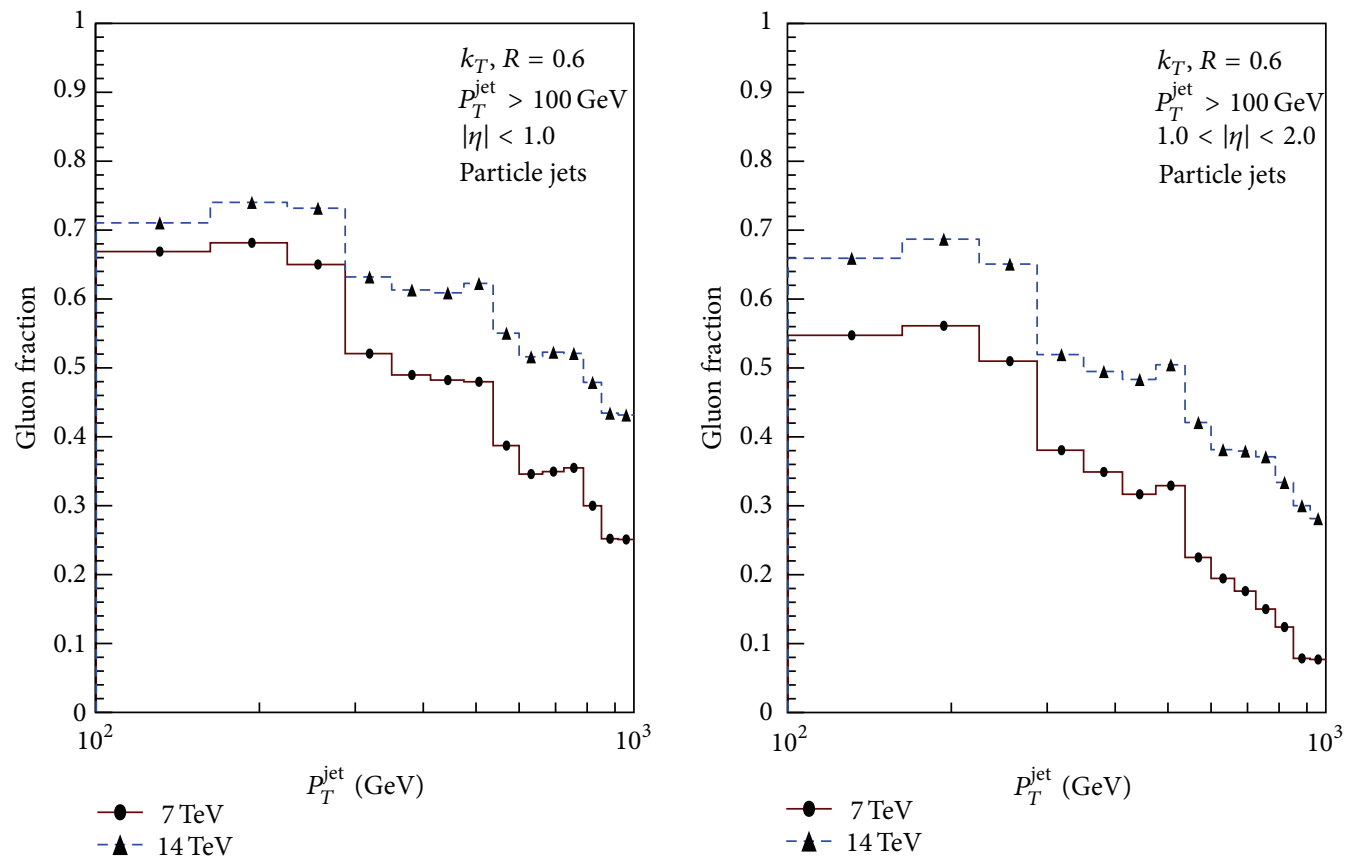

FIGURE 2: Fraction of gluon initiated jets differentiated via matching of the two particle leading jets to the hard partons of the leading-order process as a function of jet $P_{T}$ from $k_{T}$ algorithm at $\sqrt{s}=7 \mathrm{TeV}$ compared to $\sqrt{s}=14 \mathrm{TeV}$ in the pseudorapidity bins of $|\eta|<1$ and $1 \leq$ $|\eta|<2$.
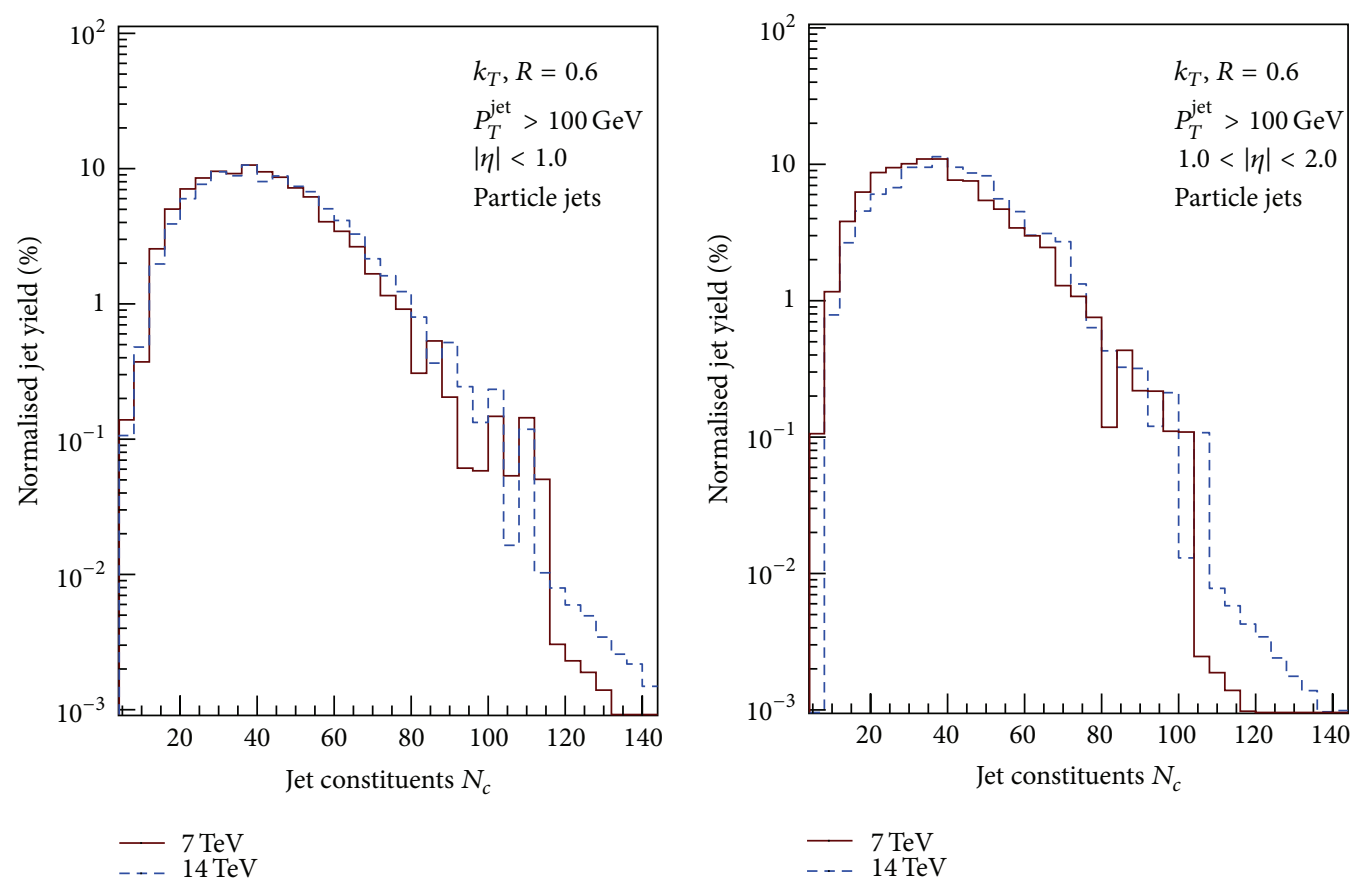

Figure 3: Distributions of the jet constituent multiplicity in particle jets having $P_{T}^{\text {jet }}>100 \mathrm{GeV}$ from $k_{T}$ algorithm at $\sqrt{s}=7$ TeV compared to $\sqrt{s}=14 \mathrm{TeV}$ in the pseudorapidity bins of $|\eta|<1$ and $1 \leq|\eta|<2$.

deviation in transverse momentum between the jet and the associated parton with respect to the jet transverse momentum is $\Delta P_{T} / P_{T}\left(\Delta P_{T} / P_{T}\right.$ is abs (parton $P_{T}-$ jet $\left.P_{T}\right) /$ jet $\left.P_{T}\right)<$ 3.0. Figure 1 shows the ratio $\Delta P_{T} / P_{T}$ for quark and gluon jets matched, respectively, to partons for $\sqrt{s}=7 \mathrm{TeV}$ and $\sqrt{s}=14 \mathrm{TeV}$. At each energy the matching of particle jets with the partons is found to be good as all the jets have the ratio less than 3 .

Figure 2 shows the fraction of gluon initiated jets via matching of the two particle jets leading in $P_{T}$ to the hard 

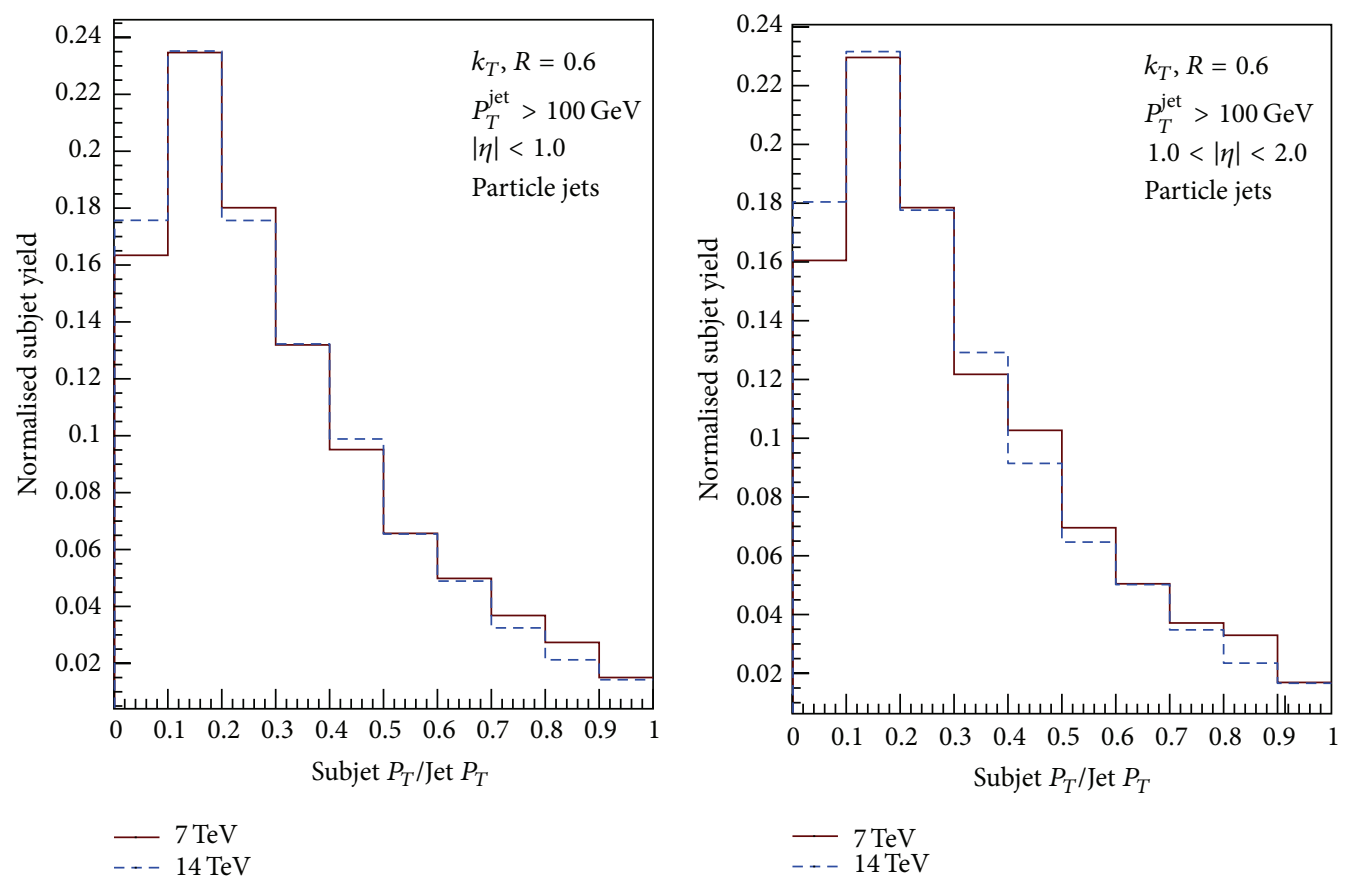

Figure 4: Distributions of the subjet $P_{T}$ fraction in particle jets having $P_{T}^{\text {jet }}>100 \mathrm{GeV}$ from $k_{T}$ algorithm at $\sqrt{s}=7 \mathrm{TeV}$ compared to $\sqrt{s}=$ $14 \mathrm{TeV}$ in the pseudorapidity bins of $|\eta|<1$ and $1 \leq|\eta|<2$.

partons of the LO process as a function of jet $P_{T}$ for the two different pseudorapidity bins, $|\eta|<1$ and $1 \leq|\eta|<2$. As expected the fraction of gluon jets decreases with increasing jet $P_{T}$ in both the cases. Also, quark jets make up a dominant fraction of the jets only at high jet transverse momenta.

\section{Systematic Uncertainties}

The uncertainties in the subjet multiplicities are of two categories:

(i) statistical uncertainties,

(ii) systematic uncertainties.

For the present analysis using Monte Carlo samples, the uncertainties are mainly on account of the statistics which are found to be negligibly small. The systematic uncertainties arise due to the change in gluon fraction due to minimum $P_{T}$ imposed. This is measured as follows.

(i) Gluon Jet fraction: The uncertainty in gluon jet fraction introduces a systematic error on the subjet multiplicity. To calculate this, the gluon jet fraction is obtained as a function of minimum jet $P_{T}(\mathrm{GeV})$ by varying minimum jet $P_{T}$ from 90 to $110 \mathrm{GeV}$. This introduces an error of \pm 0.02 on gluon fraction at $\sqrt{s}=7 \mathrm{TeV}$ and \pm 0.01 on gluon fraction at $\sqrt{s}=14 \mathrm{TeV}$. If the gluon fraction increases or decreases at both the energies, the change in ratio $r$ is not much effective. But the increase in gluon jet fraction at one energy and decrease at other or vice versa introduces a largest change in ratio $r$, giving minimum and maximum value of $r$. The difference of the calculated value $r$ given in Table 5 from the minimum value and maximum value of $r$ gives the systematic uncertainty due to gluon fraction as ${ }_{-0.05}^{+0.08}$.

The same analysis for measuring $r$, when performed on the data from a detector such as CMS at LHC, would involve the following systematic errors also. In one such measurement with $36 \mathrm{pb}^{-1}$ data used for measuring the subjet multiplicity at $\sqrt{s}=7 \mathrm{TeV}$ energy [10], we estimated the following uncertainties.

(ii) Jet-Energy calibration: The maximum systematic uncertainty in Jet-Energy calibration was estimated to be 5\% depending upon jet $P_{T}$ and jet $\eta$. This introduces an error of about $2 \%$ on the subjet multiplicity average value.

(iii) Jet-Energy resolution (JER): The uncertainty on the jet $P_{T}$ resolution (JER) was estimated to be less than $10 \%$ and this results in less than $0.25 \%$ uncertainty in the subjet multiplicity distributions.

(iv) Uncertainties due to the different physics models employed for the simulations: The uncertainty due to different physics models implemented in the Monte Carlo generators is estimated to be less than $3 \%$.

Compounding these systematic errors (ii)-(iv), the maximum uncertainty in the subjet multiplicity of 3.28 at $7 \mathrm{TeV}$ was estimated to be 0.10 . The details can be found in [10]. We use the same percent value of each of the above mentioned systematic uncertainties in subjet multiplicities at the two energies which will be reflected in ratio $r$. The systematic errors on ratio $r$ are given in Table 6 and added in quadrature 

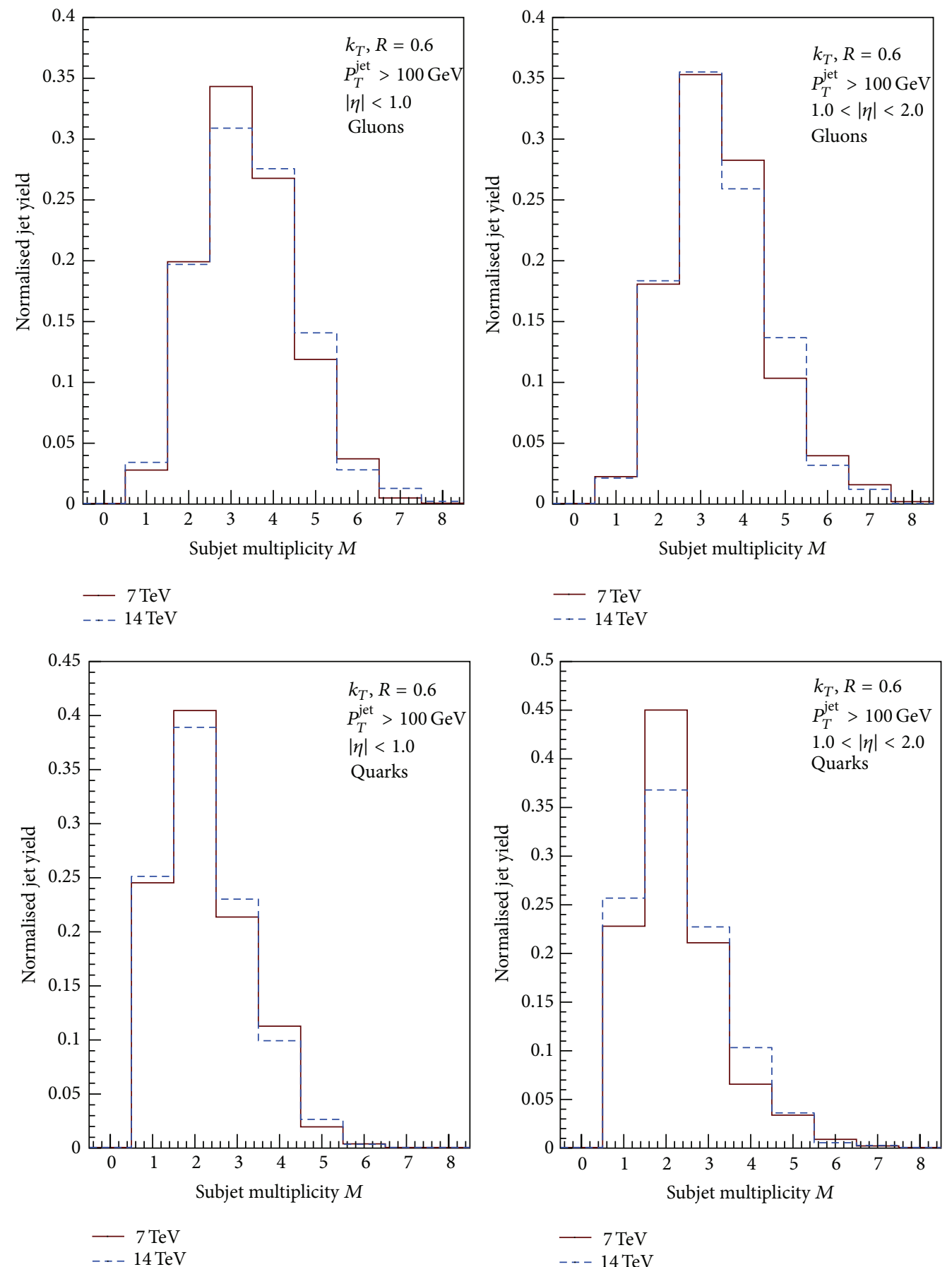

FIGURE 5: Subjet multiplicity distributions for jets matched to gluons (top) and quarks (bottom), having $P_{T}^{\text {jet }}>100 \mathrm{GeV}$, in the pseudorapidity bins of $|\eta|<1$ (left) and $1 \leq|\eta|<2$ (right) at $\sqrt{s}=7 \mathrm{TeV}$ and $\sqrt{s}=14 \mathrm{TeV}$ from $k_{T}$ algorithm.

to have the total systematic uncertainty in the calculated value of the ratio $r$.

\section{Results and Discussions}

Figure 3 shows jet constituent multiplicity distributions for $k_{T}$ algorithm at $\sqrt{s}=7 \mathrm{TeV}$ and at $\sqrt{s}=14 \mathrm{TeV}$ in the two pseudorapidity bins, $|\eta|<1$ and $1 \leq|\eta|<2$. It may be observed that the average number of jet constituents is larger at $14 \mathrm{TeV}$. The subjet $P_{T}$ fractions for the two pseudorapidity regions and for the two energies are shown in Figure 4. In $k_{T}$ algorithm, the procedure to resolve subjets is dependent on $P_{T}$ of jet and the $P_{T}$ of subjets in a particular jet is distributed in such a way that there is no sudden decrease. Thus as shown in Figure 4, we get monotonically decreasing values for subjet $P_{T}$ fraction. 
TABLE 5: Average subjet multiplicities (calculated using (7)) and ratio of subjet multiplicities (from (8)) in the phase space $|\eta|<2$ in different $P_{T}^{\text {jet }}(\mathrm{GeV})$ bins for $k_{T}$ algorithm.

\begin{tabular}{|c|c|c|c|c|c|c|}
\hline \multirow{2}{*}{$P_{T}^{\text {jet }}(\mathrm{GeV})$} & \multicolumn{2}{|c|}{ Gluon jets } & \multicolumn{2}{|c|}{ Quark jets } & \multirow{2}{*}{$r=\left\langle M_{g}\right\rangle-1 /\left\langle M_{q}\right\rangle-1$} & \multirow{2}{*}{ Stat. } \\
\hline & $\left\langle M_{g}\right\rangle$ & Stat. & $\left\langle M_{q}\right\rangle$ & Stat. & & \\
\hline $100-300$ & 3.17 & \pm 0.18 & 1.90 & \pm 0.17 & 2.42 & \pm 0.25 \\
\hline $300-500$ & 2.39 & \pm 0.06 & 1.75 & \pm 0.05 & 1.87 & \pm 0.07 \\
\hline $500-700$ & 2.33 & \pm 0.04 & 1.56 & \pm 0.03 & 2.36 & \pm 0.06 \\
\hline $700-900$ & 2.25 & \pm 0.04 & 1.52 & \pm 0.03 & 2.42 & \pm 0.06 \\
\hline $900-1100$ & 2.19 & \pm 0.04 & 1.49 & \pm 0.02 & 2.43 & \pm 0.06 \\
\hline
\end{tabular}

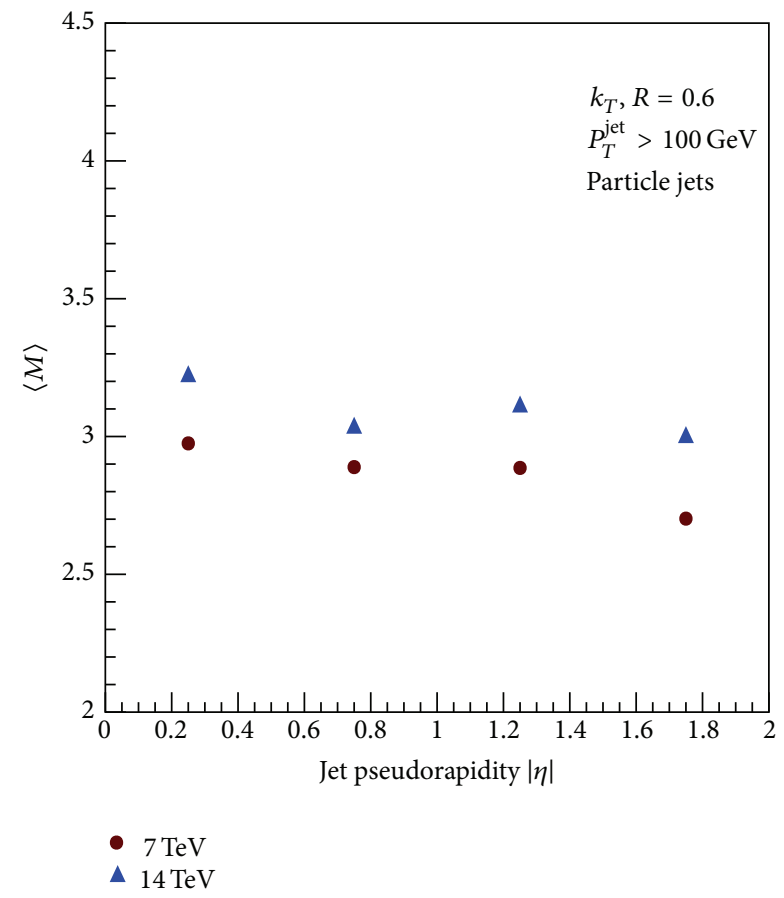

FIGURE 6: Average subjet multiplicity $\langle M\rangle$ as a function of absolute jet pseudorapidity $|\eta|$ for the whole range in $\widehat{p}_{T}$ at $\sqrt{s}=7 \mathrm{TeV}$ and $\sqrt{s}=14 \mathrm{TeV}$ for $k_{T}$ algorithm.

Figure 5 shows the subjet multiplicity distributions for jets matched to gluons in the two pseudorapidity bins and jets matched to quarks in the two pseudorapidity bins $|\eta|<1$ and for $1 \leq|\eta|<2$. The mean values are given in Table 1 . Average number of subjets in gluon jets is found to be larger than in the quark jets indicating a higher degree of collimation in quark jets.

Average subjet multiplicity, $\langle M\rangle$ dependence on absolute jet pseudorapidity for $\sqrt{s}=7 \mathrm{TeV}$ and at $\sqrt{s}=14 \mathrm{TeV}$ for the whole $\widehat{p}_{T}$ range, is shown in Figure 6. Figure 7 shows the subjet multiplicity distributions of jets as a function of the pseudorapidity bins $(0.0-0.5,0.5-1.0,1.0-1.5$, and $1.5-2.0)$ for four $\widehat{p}_{T}$ intervals $50-300,300-550,550-800$, and $800-$ $1050 \mathrm{GeV}$. The values are given in Table 2. We observe that from inner to outer rapidities windows, with same rapidity
TABLE 6: Systematic uncertainties on ratio of subjet multiplicities from different sources.

\begin{tabular}{lc}
\hline Source & $\delta \mathrm{r}$ \\
\hline Gluon jet fraction & $(+0.08)$ \\
Jet-energy calibration & $(-0.05)$ \\
Jet-energy resolution & \pm 0.15 \\
Different physical model & \pm 0.02 \\
\hline \multirow{2}{*}{ Total } & \pm 0.22 \\
\hline
\end{tabular}

window size, the subjet multiplicity tends to get smaller very slightly.

Figure 8 shows the jet $P_{T}$ versus $\langle M\rangle$ for pseudorapidity $|\eta|<2$ for quarks, gluons, and matched jets for the two energies under study. The mean values are given in Table 3.

The selected dijet sample consists of mixture of gluon and quark initiated jets as in the LO hard QCD $2 \rightarrow$ 2 processes. Following the recipe from Section 3, we use (7) to separate these two types and obtain the average subjet multiplicities $\langle M\rangle$ for gluon jets and quark jets in the five bins of jet $P_{T}$. The results are given in Table 5 (the weighted mean $\bar{M}$ and the uncertainties are calculated by using $\bar{M}=\left(\sum M_{i} / \operatorname{error}_{i}^{2}\right) /\left(\sum 1 /\right.$ error $\left._{i}^{2}\right)$ and $\operatorname{error}_{\bar{M}}^{2}=$ $1 /\left(\sum 1 /\right.$ error $\left.\left._{i}^{2}\right)\right)$. These values are then used to calculate the ratio $r$ from (8) as given in Table 5.

\section{Summary}

The subjet multiplicities in proton-proton collisions at $\sqrt{s}=$ $7 \mathrm{TeV}$ and at $\sqrt{s}=14 \mathrm{TeV}$ are estimated in 14 million protonproton collisions by selecting dijet samples simulated using PYTHIA8.

The jets and subjets have been resolved with the $k_{T}$ jet algorithm for a jet size of $R=0.6$ and a subjet resolution cutoff $y_{\text {cut }}=10^{-3}$. The subjet multiplicity distribution and its average $\langle M\rangle$ have been determined in the jet $P_{T}$ range of $100-1100 \mathrm{GeV}$ for jet pseudorapidity $|\eta|<2$. In the whole pseudorapidity range $|\eta|<2$, the average subjet multiplicity $\langle M\rangle$ for particle jets is found to decrease from $2.63 \pm 0.01$ (stat.) down to $1.64 \pm 0.01$ (stat.) for $7 \mathrm{TeV}$ and from $2.76 \pm 0.01$ (stat.) down to $1.75 \pm 0.01$ (stat.) at $14 \mathrm{TeV}$ with increasing jet $P_{T}$. The average subjet multiplicity for jets 

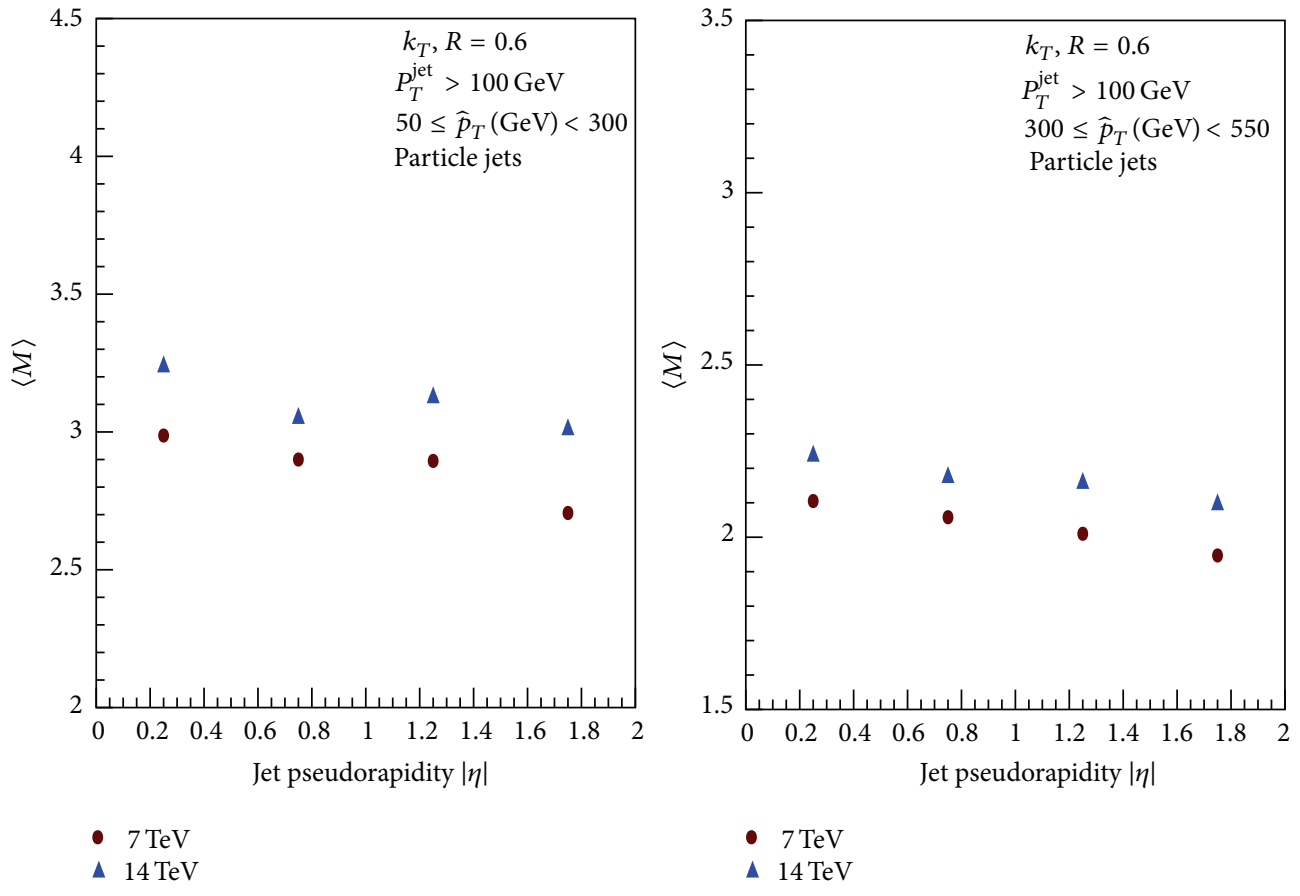

- $7 \mathrm{TeV}$

- $14 \mathrm{TeV}$
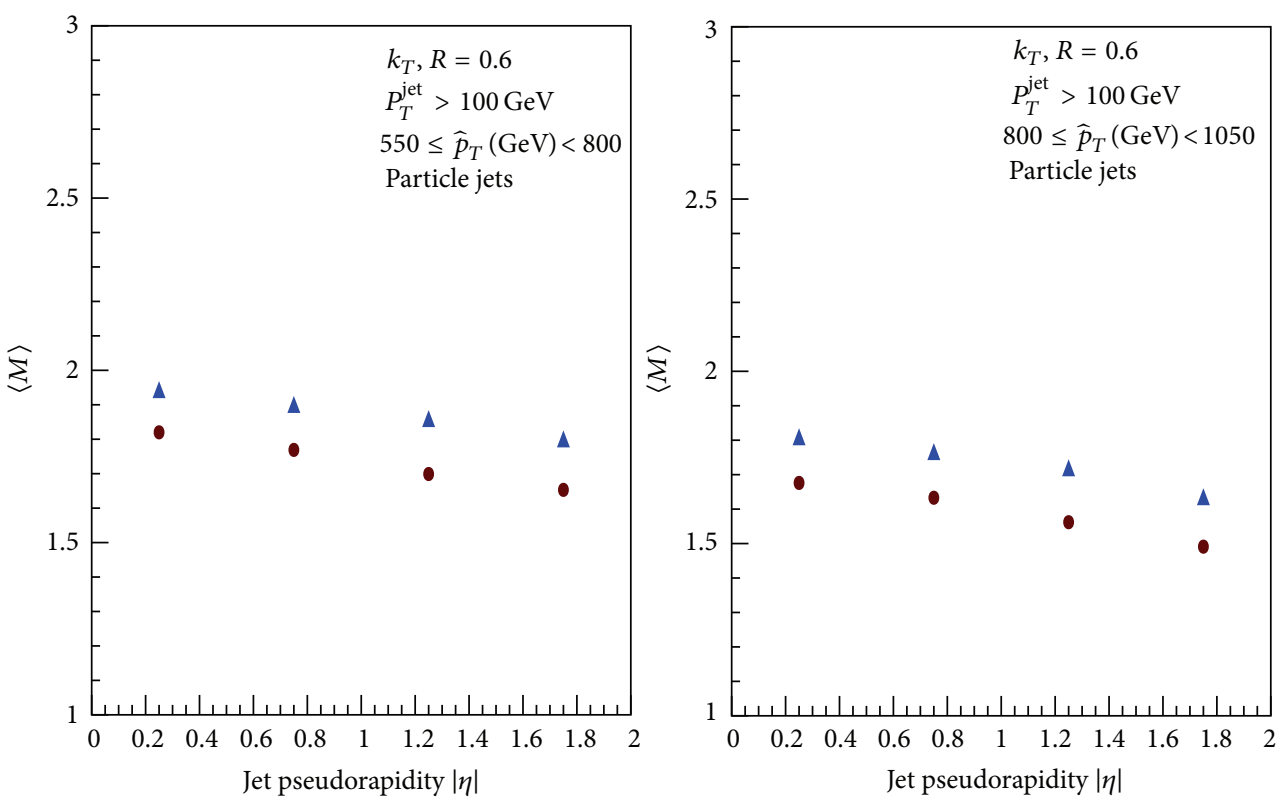

- $7 \mathrm{TeV}$

- $7 \mathrm{TeV}$

A $14 \mathrm{TeV}$

\ $14 \mathrm{TeV}$

FIGURE 7: Average subjet multiplicity $\langle M\rangle$ as a function of absolute jet pseudorapidity $|\eta|$ in the four $\widehat{p}_{T}$ bins at $\sqrt{s}=7 \mathrm{TeV}$ and $\sqrt{s}=14 \mathrm{TeV}$ for $k_{T}$ algorithm.

matched to gluons, $\left\langle M_{g}\right\rangle$, is also found to decrease from $3.06 \pm 0.01$ (stat.) down to $2.11 \pm 0.01$ (stat.) for $7 \mathrm{TeV}$ and from $3.07 \pm 0.01$ (stat.) down to $2.11 \pm 0.01$ (stat.) at $14 \mathrm{TeV}$ with increase in jet $P_{T}$ from $100 \mathrm{GeV}$ to $1100 \mathrm{GeV}$. The average subjet multiplicity for jets matched to quarks, $\left\langle M_{q}\right\rangle$, is also found to decrease from $2.05 \pm 0.01$ (stat.) down to $1.49 \pm 0.01$ (stat.) for $7 \mathrm{TeV}$ and from $2.13 \pm 0.01$ (stat.) down to $1.52 \pm 0.01$ (stat.) at $14 \mathrm{TeV}$ with increase in jet $P_{T}$.
This is a clear indication of a higher degree of collimation with rising jet $P_{T}$. In Table 2, we can observe that from inner to outer rapidities the subjet multiplicity tends to get slightly smaller as well. Evidently, a direct conclusion on the gluon-quark fraction cannot be drawn since the subjet multiplicity not only depends on this fraction but also on details of the Multiple Parton Interactions (MPI), parton shower, and hadronization models. Exploiting data at two 
TABLE 7: Color factor ratio for different experiments.

\begin{tabular}{lccr}
\hline Experiment & Ratio $C_{A} / C_{F}$ & Stat. & Sys. \\
\hline Dø (2 jet events) [12] & 1.91 & \pm 0.04 & \pm 0.21 \\
DELPHI (3 jet events) [13] & 1.74 & \pm 0.03 & \pm 0.10 \\
OPAL (4 jet events) [14] & 2.11 & \pm 0.16 & \pm 0.28 \\
DELPHI (4 jet events) [15] & 2.12 & \pm 0.35 & \pm 0.11 \\
ALEPH (4 jet events) [16] & 2.20 & \pm 0.09 & \pm 0.13 \\
\hline
\end{tabular}

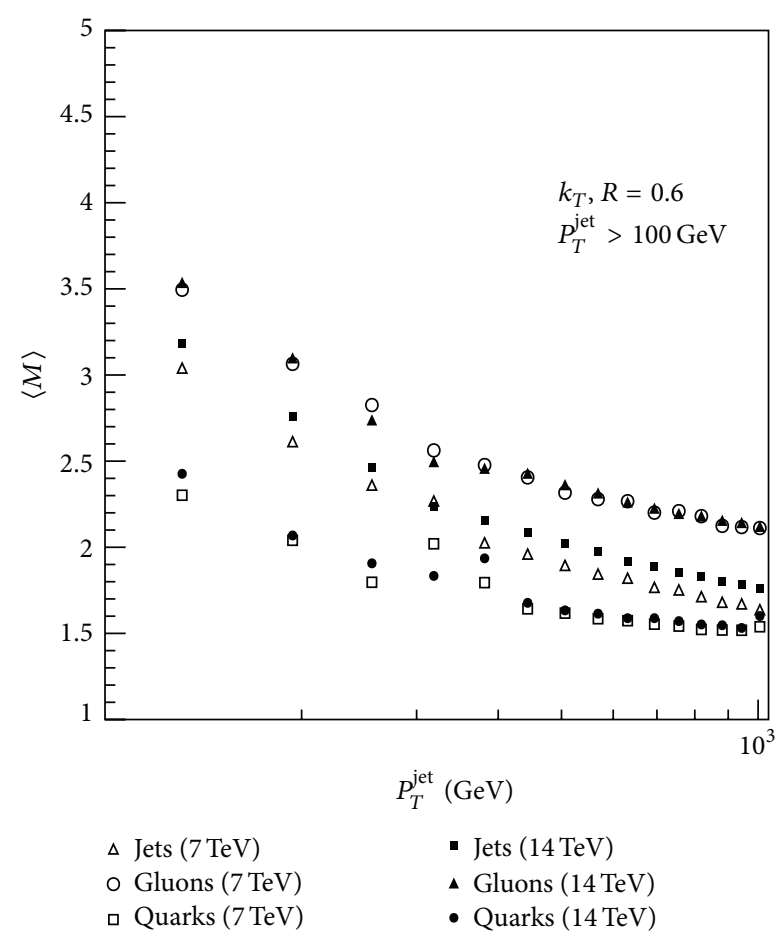

FIGURE 8: Average subjet multiplicity $\langle M\rangle$ as a function of jet $P_{T}$ in the pseudorapidity range of $|\eta|<2$ at $\sqrt{s}=7 \mathrm{TeV}$ and $\sqrt{s}=14 \mathrm{TeV}$ for $k_{T}$ algorithm.

different center-of-mass energies, the model dependence can be potentially eliminated. This technique was applied for data at two center-of-mass energies at Tevatron, as described in [12] to measure the ratio $r$. This method has been exploited for present and future LHC energies to predict the value of ratio $r$. The values of average subjet multiplicities in quark and gluon jets in different jet $P_{T}$ bins are calculated from (7) using the values given in Tables 3 and 4 and the ratio from (8). The results are given in Table 5. The mean value of ratio $r$ is calculated by using maximum likelihood estimator of the subjet distributions as $r=2.30 \pm 0.03$ (stat.). The total systematic uncertainty from different sources comes out to be ${ }_{-0.27}^{+0.28}$, given in Table 6 . So the extracted value of ratio of subjet multiplicities of gluon jets and quark jets is $r=2.30 \pm$ 0.03 (stat.) ${ }_{-0.27}^{+0.28}$ (sys.). This value converges to the ratio of the color factors, $C_{A} / C_{F}=9 / 4$. In [17], it is shown that the ratio of the multiplicities from quark and gluon jets should converge to the color factor ratio. The extracted value is in good agreement with the value predicted from the symmetry group SU(3) of QCD and also with the values measured at previous experiments listed in Table 7.

\section{Conflict of Interests}

The authors declare that there is no conflict of interests regarding the publication of this paper.

\section{Acknowledgment}

The authors would like to acknowledge Klaus Rabbertz for his contributions in steering the work reported in [14, 15] from where the estimation of some errors has been adopted in the present paper.

\section{References}

[1] R. K. Ellis, W. J. Stirling, and B. R. Webber, QCD and Collider Physics, Cambridge University Press, Cambridge, UK, 1996.

[2] G. P. Salam, "Towards Jetography," The European Physical Journal C, vol. 67, no. 3-4, pp. 637-686, 2010.

[3] S. Catani, Y. L. Dokshitzerc, M. Olssond, G. Turnocka, and B. R. Webbera, "New clustering algorithm for multijet cross sections in $\mathrm{e}^{+} \mathrm{e}^{-}$annihilation," Physics Letters B, vol. 269, no. 3-4, pp. 432-438, 1991.

[4] S. Catani, Y. L. Dokshitzer, and B. R. Webber, "The $k_{\perp}$-clustering algorithm for jets in deep inelastic scattering and hadron collisions," Physics Letters B, vol. 285, no. 3, pp. 291-299, 1992.

[5] S. D. Ellis and D. E. Soper, "Successive combination jet algorithm for hadron collisions," Physical Review D, vol. 48, no. 7, pp. 3160-3166, 1993.

[6] M. Cacciari, G. P. Salam, and G. Soyez, "FastJet user manual," The European Physical Journal C, vol. 72, 2012.

[7] M. Cacciari, G. P. Salam, and G. Soyez, "FastJet homepage," http://www.lpthe.jussieu.fr/salam/fastjet/ .

[8] M. Cacciari and G. P. Salam, "Dispelling the $N^{3}$ myth for the $k_{T}$ jet-finder," Physics Letters B, vol. 641, no. 1, pp. 57-61, 2006.

[9] T. Sjostrand, S. Mrenna, and P. Z. Skands, "A brief introduction to PYTHIA 8.1," Computer Physics Communications, vol. 178, no. 11, pp. 852-867, 2008.

[10] The CMS Collaboration, "Measurement of the Subjet Multiplicity in Dijet Events from proton-proton collisions at $\sqrt{s}=7 \mathrm{TeV}$," CMS PAS QCD-10-041, 2012.

[11] M. Zubin Mehta, M. Kaur, K. Rabbertz et al., "Measurement of the Subjet Multiplicity in Dijet Events from proton-proton collisions at $\sqrt{s}=7 \mathrm{TeV}$," CMS AN-2010-252, 2011. 
[12] DØ Collaboration, "Subjet multiplicity of gluon and quark jets reconstructed with the $k_{\perp}$ algorithm in $\rho \bar{\rho}$ fip collisions," Physical Review D, vol. 65, no. 5, Article ID 052008, 20 pages, 2002.

[13] J. William Gary, "Determination of the QCD color factor ratio $C_{A} / C_{F}$ from the scale dependence of multiplicity in three jet events," Physical Review D, vol. 61, no. 11, Article ID 114007, 8 pages, 2000.

[14] R. Akers, G. Alexander, J. Allison et al., "A measurement of the QCD colour factor ratios $C_{A} / C_{F}$ and $T_{F} / C_{F}$ from angular correlations in four-jet events," Zeitschrift für Physik C, vol. 65, no. 3, pp. 367-377, 1995.

[15] P. Abreu, W. Adam, T. Adye et al., "Measurement of the triplegluon vertex from 4-jet events at LEP," Zeitschrift für Physik C, vol. 59, no. 3, pp. 357-368, 1993.

[16] The ALEPH Collaboration, "A measurement of the QCD colour factors and a limit on the light gluino," Zeitschrift für Physik C, vol. 76, pp. 1-14, 1997.

[17] E. D. Malaza, "Multiplicity distributions in quark and gluon jets to $O(\alpha)_{s}$," Zeitschrift für Physik C, vol. 31, no. 1, pp. 143-150, 1986. 

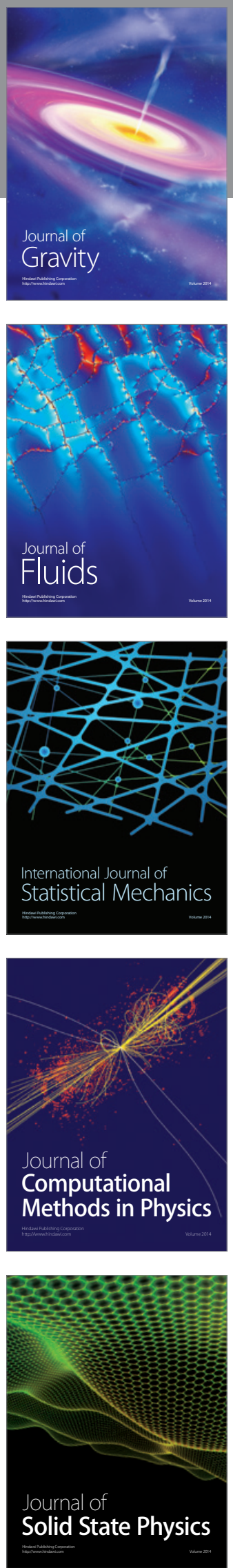

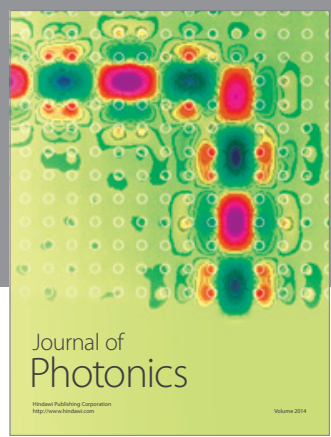

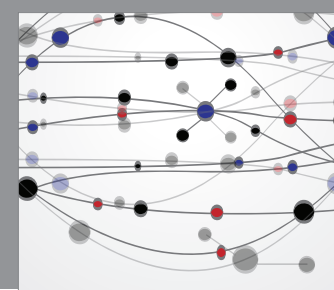

The Scientific World Journal

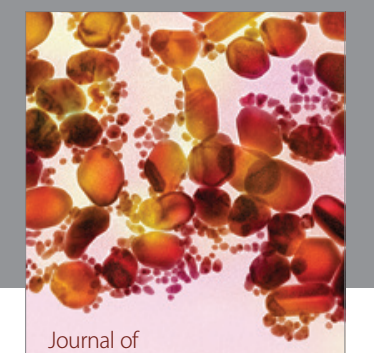

Soft Matter
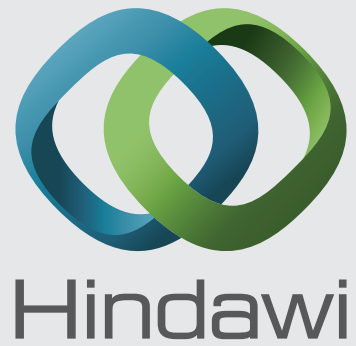

Submit your manuscripts at

http://www.hindawi.com
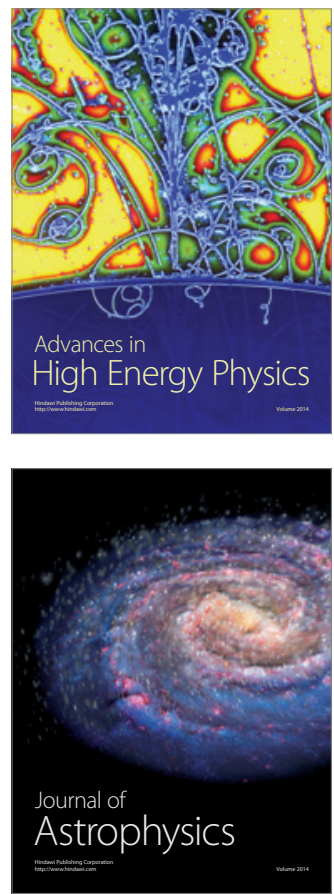
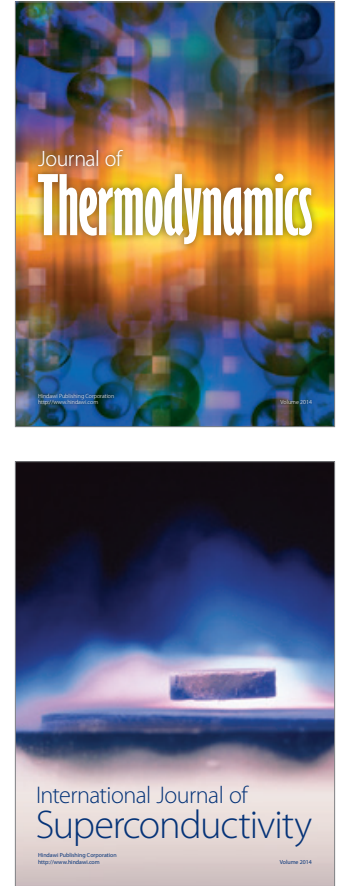
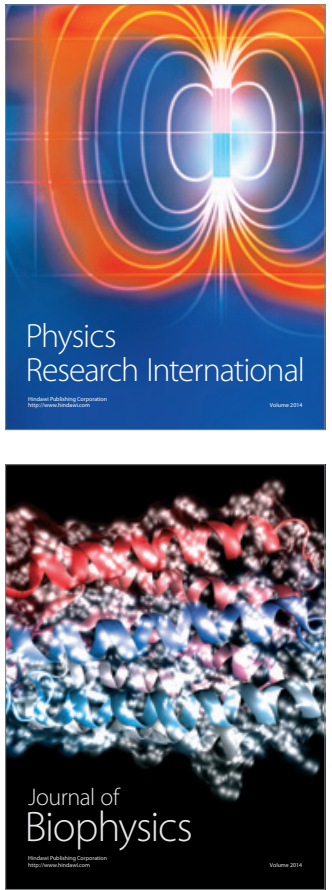
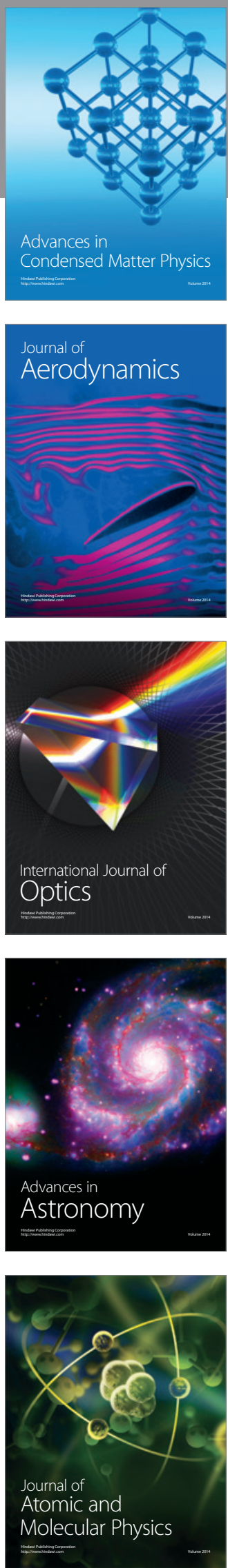